
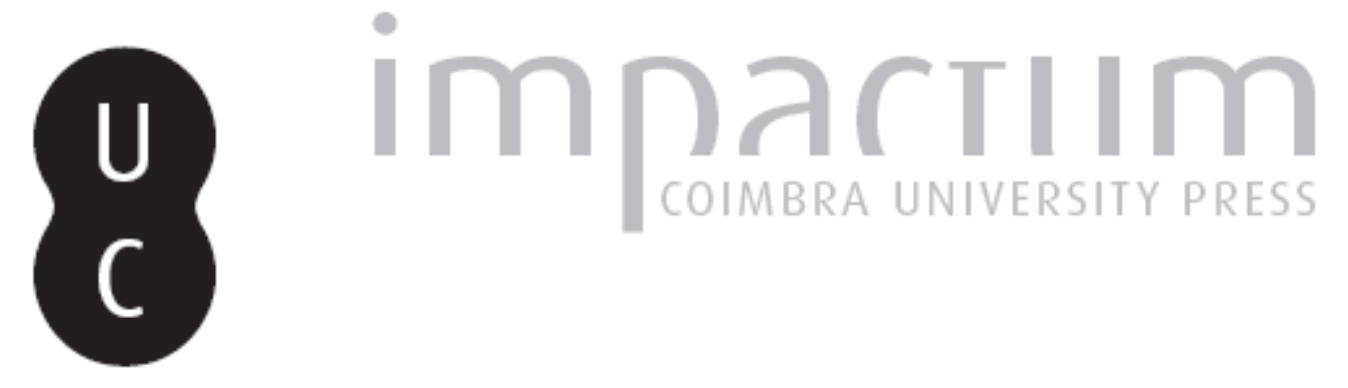

La scenografia come architettura: Niccolò Nasoni e la Torre dos Clérigos

Autor(es): $\quad$ Raggi, Giuseppina

Publicado por: Imprensa da Universidade de Coimbra

URL persistente:

URI:http://hdl.handle.net/10316.2/42432

DOI:

DOl:https://doi.org/10.14195/0870-8584_8_8

Accessed : $\quad$ 26-Apr-2023 14:09:33

A navegação consulta e descarregamento dos títulos inseridos nas Bibliotecas Digitais UC Digitalis, UC Pombalina e UC Impactum, pressupõem a aceitação plena e sem reservas dos Termos e Condições de Uso destas Bibliotecas Digitais, disponíveis em https://digitalis.uc.pt/pt-pt/termos.

Conforme exposto nos referidos Termos e Condições de Uso, o descarregamento de títulos de acesso restrito requer uma licença válida de autorização devendo o utilizador aceder ao(s) documento(s) a partir de um endereço de IP da instituição detentora da supramencionada licença.

Ao utilizador é apenas permitido o descarregamento para uso pessoal, pelo que o emprego do(s) título(s) descarregado(s) para outro fim, designadamente comercial, carece de autorização do respetivo autor ou editor da obra.

Na medida em que todas as obras da UC Digitalis se encontram protegidas pelo Código do Direito de Autor e Direitos Conexos e demais legislação aplicável, toda a cópia, parcial ou total, deste documento, nos casos em que é legalmente admitida, deverá conter ou fazer-se acompanhar por este aviso. 


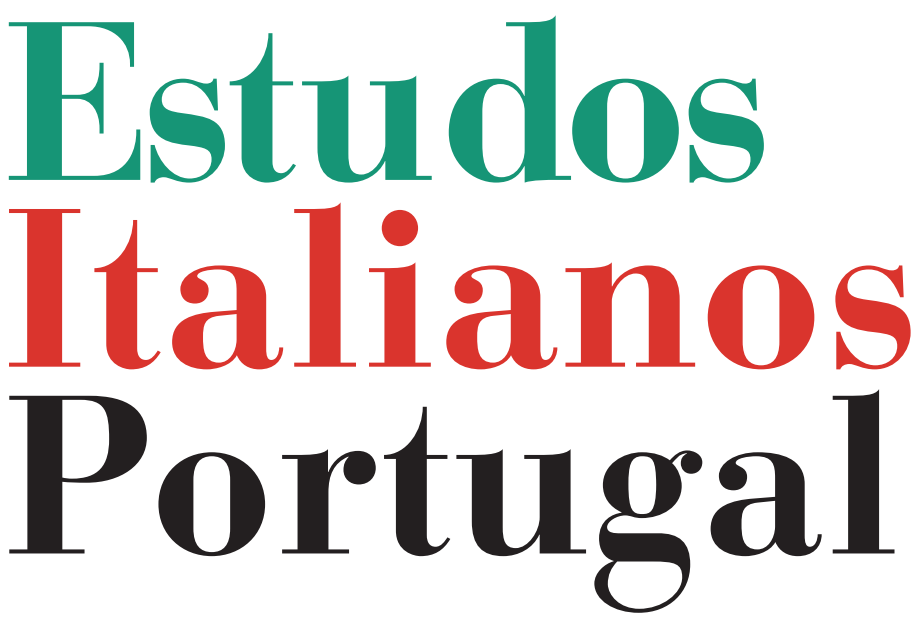

Instituto

Italiano

de Cultura

de Lisboa

Nova Série

$\mathbf{N}^{\circ} \mathbf{8}$ 


\section{LA SCENOGRAFIA COME ARCHITETTURA: NICCOLÒ NASONI E LA TORRE DOS CLÉRIGOS}

GiUSEPPINA RAGGI*

LA CELEBRAZIONE DEL $250{ }^{\circ}$ anniversario della torre campanaria della chiesa di São Pedro dos Clérigos di Porto, la cui costruzione fu conclusa nel 1763, mi ha offerto l'occasione di rivisitare alcuni miei studi sulla formazione e l'opera di Niccolò Nasoni e di integrarli con nuove riflessioni alla luce dei più recenti contributi critici. In questo breve testo tenterò di delineare i motivi che giustificano la nota espressione di Robert Smith riferita alle opere progettate da Niccolò Nasoni come "uma arquitectura de festival perene" ${ }^{1}$. E' importante considerare che, quando il critico americano la coniò, il periodo di formazione bolognese dell'artista rimaneva ancora sconosciuto agli studiosi, così come veniva sottostimata l'influenza della pratica scenografica nell'ambito del costruito. Robert Smith ricercava le fonti dell'opera di Nasoni tra i modelli dell'architettura borrominiana, avanzando un parallelismo stilistico con il barocco leccese che, sino ad ora, non è stato comprovato da alcun dato documentario. L'efficacia

* Ricercatrice integrata del Centro de História de Além-mar dell'Universidade Nova de Lisboa. Dottore di ricerca in Storia dell'Arte, dall'Universidade de Lisboa e dall'Università degli Studi di Bologna, si dedica allo studio della quadratura e ai rapporti artistici tra l'Italia e il mondo portoghese durante il XVII e XVIII secolo.

${ }^{1}$ Robert Smith, "Nicolau Nasoni (1691-1773)”, Bracara Augusta, 28, 65-66 (7778), 1974, p. 10. 
evocativa della definizione, però, è indubbia e se ai rapporti con l'architettura romana dell'epoca si aggiungono quelli della quadratura e della scenografia, la peculiarità stilistica del nord del Portogallo, marcata profondamente dall'attività di Nasoni, acquista un aspetto più coerente all'interno del panorama europeo settecentesco.

Nato a San Giovanni Valdarno nel 1691, Niccolò Nasoni svolge gli anni italiani della sua formazione e della sua carriera d'artista in stretta connessione con le vicende politicoartistiche della città di Siena e con le committenze delle più potenti famiglie locali. Nella città toscana, infatti, consolida il suo apprendistato, instaurando un legame duraturo con Vincenzo Ferrati, scenografo dell'Accademia dei Rozzi, di cui assume la funzione nel $1711^{2}$.

La prima maturità di Nasoni si sviluppa dunque in ambito toscano. Egli si dedica alla pratica scenografica e alla realizzazione di architetture effimere in quanto membro della citata Accademia, che, come istituzione preposta all'attività teatrale, s'incaricava anche di allestire la città in occasione di feste e entrate solenni. Opera anche come quadraturista, affiancando il pittore figurista Giuseppe Nasini negli affreschi dipinti

${ }^{2}$ Vedi Niccolò Nasoni (1691-1773). Un artista italiano a Oporto. Catalogo della mostra, Firenze, Ponte delle Grazie, 1991, 2 voll.; Giuseppina Raggi, "A formação bolonhesa de Niccolò Nasoni: algumas antecipações", Monumentos, 14, Março 2001, pp. 33-41; id., "La geografia artistica di Niccolò Nasoni, quadraturista-scenografo girovago", Commentari d'Arte, 18-19, 7, 2001, pp. 49-56; id., "Il viaggio delle forme: la diffusione della quadratura nel mondo portoghese del Settecento", Fauzia Farneti, Deanna Lenzi, L'architettura dell'Inganno. Quadraturismo e grande decorazione nella pittura di età barocca. Atti del convegno internazionale di studi, Rimini, novembre 2002, Firenze, Alinea, 2004, pp. 161-174; Giuseppina Raggi, "Le fonti bolognesi della quadratura luso-brasiliana: la formazione di Niccolò Nasoni e di Vincenzo Bacherelli", Arte a Bologna, 06, 2007, pp. 46-55; Giovanni Battista Tedesco, "Niccolò Nasoni: studio sugli affreschi della capela-mor nella Sé Catedral di Oporto", Revista da Faculdade de Letras. Ciência e Técnica do Patrimônio, I s., vol. 5-6, 2006-2007, pp. 587-600; Giovanni Battista Tedesco, Nicolau Nasoni: formação de um pintor e de um artista da arte efémera em Itália (1691-1723), tese de doutoramento, Universidade de Porto, 2011. 
a palazzo Cennini tra il 1714 e il $1715^{3}$. Nel 1715 progetta l'arco trionfale innalzato per festeggiare l'entrata del nuovo vescovo della città Alessandro Zondadari. In calce al disegno che ne conserva la memoria si legge:

Arco di ordine dorico dipinto a chiaroscuro ed a due facce, alto braccia 24, eretto dall'Accademia de' Rozzi sull'ingresso della piazza del Duomo, d'invenzione e fattura di Niccolò Nasoni Pittore Senese, e lineato quivi dal Signor Giovanni Franchini pubblico Architetto, in occasione del solenne possesso di Mons.r Alessandro Zondadari ${ }^{4}$

Il disegno dimostra il rispetto delle proporzioni e delle parti proprie all'ordine architettonico prescelto. A coronamento dell'architrave un basamento lineare sostiene le allegorie della Fortezza e della Giustizia che si ergono, in forma di statue, ai lati dello scudo araldico della famiglia ChigiZondadari che troneggia al centro. Il modello grafico non corrisponde integralmente al medesimo apparato descritto nella Biografia cronologia dè bellartisti senesi: 1200-1800, così ricordato:

col disegno ed esecuzione del nostro artista [Nasoni] i Sigri dell'Accademia dei Rozzi fecero il superbo apparato per tutta la strada del capitano presso il palazzo Reale, adornandolo di statue dipinte figuranti la Gloria, Liberalità, Musica, Matematica, Poesia, Pittura, Scultura, Architettura, Geografia, Aritmetica e Retorica. Nel terminare di detta strada dalla parte della piazza del Duomo fu eretto un magnifico arco trionfale d'ordine dorico con le virtù cardinali situate in varie nicchie e in alto una gigantesca figura rappresentando la fama .

${ }^{3}$ La biografia senese di Nasoni è riportata da Ettore Romagnoli, Biografia cronologia dè bellartisti senesi: 1200-1800, Firenze, S.P.E.S., 1976 (facsimile del manoscritto del 1835).

${ }^{4}$ Siena, Biblioteca degli Intronati, inv. S. III.6/25.

${ }^{5}$ Ettore Romagnoli, Biografia cronologia dè bellartisti senesi, vol. 12, p. 45. 
L'arco viene considerato "il più bello di tutti" e e il successo riscosso in questa occasione rappresenta l'evento propiziatorio del soggiorno bolognese dell'artista, grazie all'apprezzamento e alla protezione da parte della potente famiglia senese dei Chigi-Zondadari. Questo ramo venne creato alla morte del cardinal Flavio Chigi (1631-1693), nipote di Alessandro VII, Fabio Chigi (1599-1667). Flavio lasciò per testamento il cognome e le armi della famiglia Chigi al nipote Bonaventura (1652-1719), figlio della sorella Agnese Chigi e di Ansano Zondadari. La biografia di Nasoni si intreccia con quelle dei fratelli di Bonaventura Chigi-Zondadari: Alessandro (16701745) che fu vescovo di Siena e per il quale progetta l'allestimento dell'entrata solenne; Antonio Felice (1665-1737), elevato alla dignità cardinalizia, fu legato pontificio di Bologna nei primi anni del XVIII secolo; Marcantonio (1658-1722) fu eletto gran maestro dell'Ordine dei Cavalieri di Malta nel 1720. Per quest'ultima occasione Niccolò progetta i carri di Marte e di Minerva utilizzati durante i festeggiamenti senesi e dei quali si conservano i disegni ${ }^{7}$. Niccolò Nasoni lavora a stretto contatto con architetti e stuccatori senesi, quali Jacopo Franchini e Bartolomeo e Pietro Cremoni, che uniscono alla tradizione toscana le novità borrominiane. Un soggiorno romano, ancora da comprovare su base documentaria, appare plausibile in questi anni, considerando che la sua presenza a Siena si fa più rarefatta a partire dal 1716 e che in questi anni i bolognesi Stefano Orlandi e Giovanni Orsoni lavorano a Lucca e, soprattutto, nei teatri della capitale pontificia. ${ }^{8}$ Il loro incontro potrebbe essersi verificato a Roma e, in ogni

${ }^{6}$ Niccolò Nasoni (1691-1773), p. 26.

${ }^{7}$ Siena, Biblioteca degli Intronati, Pecci, Miscellanee, ms. A.III.3, tomo I, c. 84. Vedi anche Niccolò Nasoni (1691-1773), p. 27; Giuseppina Raggi, "Le fonti bolognesi della quadratura luso-brasiliana: la formazione di Niccolò Nasoni e di Vincenzo Bacherelli”, p. 49, fig. 3.

${ }^{8}$ Elisabetta Landi, "Stefano orlandi (1681-1760)", Il Carrobbio, 7, 1981, pp. 208217. 
caso, nel 1718, Niccolò Nasoni si ritrova stabilmente a Bologna presso la "scuola dell'Orlandi", mantenendo constanti, nel frattempo, i rapporti con Siena.

Le fonti storiografiche locali hanno conservato la memoria del suo passaggio nella città emiliana e il loro rinvenimento ha aggiunto un tassello fondamentale per la comprensione delle opere architettoniche realizzate a Oporto ${ }^{10}$. Così, infatti, lo ricorda Marcello Oretti:

Nicola Nasoni, pittore senese di architettura, venne a Bologna per fare li suoi studi nell'Architettura, e godè la buona sorte di essere ammesso alla scuola di Stefano Orlandi, valoroso maestro di que' tempi; non perdè tempo dunque e fece profitto con li insegnamenti di un così amato precettore, perché apprese un franco disegno ed un vago colorito, quale era la dote più preggiata dell'Orlandi. Si trattenne in Bologna parecchi anni, ne poi ho avuto altro incontro di opere sue dopo la partenza da questo paese [...] Il d $\mathrm{d}^{\circ}$ Niccolò andò in Malta e dipinse la chiesa di S. Giovanni Battista ed altre molte fatture ${ }^{11}$.

Gli anni bolognesi segnano un momento marcante per la traiettoria artistica di Nasoni. La città era il centro europeo maggiormente specializzato nei campi della scenografia e della quadratura. Sin dalla prima metà del Seicento Agostino Mitelli e Angelo Michele Colonna avevano reinventato la pittura ad affresco di architetture (la quadratura, appunto $)^{12}$,

${ }^{9}$ Bologna, Biblioteca Comunale dell'Archiginnasio, sezione manoscritti, Marcello Oretti, Notizie dei professori del Disegno, cioè dei Pittori, Scultori ed Architetti bolognesi e dé forestieri di quella scuola, ms. B.132, c. 199.

${ }^{10}$ Giuseppina Raggi, "A formação bolonhesa de Niccolò Nasoni: algumas antecipaçôes"; id., "La geografia artistica di Niccolò Nasoni, quadraturista-scenografo girovago" .

${ }^{11}$ Bologna, Biblioteca Comunale dell'Archiginnasio, sezione manoscritti, Marcello Oretti, Notizie dei professori del Disegno, cioè dei Pittori, Scultori ed Architetti bolognesi e dé forestieri di quella scuola, ms. B.132, c. 199.

${ }^{12}$ Per una sintesi recente vedi António Filipe Pimentel, Giuseppina Raggi, David Garcia Cueto, Ilusionismos. Os Tetos Pintados do Palácio Alvor. Catálogo da exposição, MNAA, Março-Maio 2013, Lisboa, MNAA, 2013. 
così come Ferdinando e Francesco Bibiena, tra XVII e XVIII secolo, avevano sancito l'abilità in campo prospettico della scuola emiliana innovando la scenografia. Pur non facendo ancora parte del corpo docente dell'Accademia Clementina, fondata nel 1711, Stefano Orlandi era uno dei pittori-scenografi di maggior spicco della prima metà del Settecento. Come prevedeva la prassi formativa degli artisti bolognesi, oltre all'apprendimento con il maestro, Niccolò Nasoni si dedica a copiare graficamente architetture costruite o dipinte nel secolo precedente. All'interno di inevitabili oscillazioni, la scuola bolognese del Settecento rimase sempre attenta alla lezione dei suoi maestri, in particolare delle quadrature di Mitelli e Colonna, così come delle soluzioni più scenografiche dell'architettura emiliana realizzate nella seconda metà del secolo.

Alla luce dei nuovi contributi critici sullo stretto rapporto professionale e personale tra Vincenzo Ferrati e Niccolò Nasoni ${ }^{13}$ si suffraga l'ipotesi, da me avanzata nel 2001 ${ }^{14}$, che i due album di disegni custoditi alla biblioteca di Siena e attribuiti rispettivamente a ciascun artista siano piuttosto miscellanee di entrambi ${ }^{15}$. Nell'album di Ferrati si ritrovano, infatti, alcuni disegni di opere bolognesi che richiedono la presenza in loco di colui che le ha schizzate. Con mano rapida e tracciati a lapis nero si individua lo studio della quadratura della cappella del Rosario nella chiesa di San Domenico, realizzata da Mitelli e Colonna tra il 1654 e il 1656, e quello dello scalone nobile di palazzo Ranuzzi ${ }^{16}$. Questi ed altri fo-

${ }^{13}$ Giovanni Battista Tedesco, Nicolau Nasoni: formação de um pintor e de um artista da arte efémera em Itália (1691-1723).

${ }^{14}$ Giuseppina Raggi "La geografia artistica di Niccolò Nasoni, quadraturista-scenografo girovago", p. 51.

${ }^{15}$ Siena, Biblioteca degli Intronati, Ciaccheri, Disegni di Vincenzo Ferrati, S. II.1; Ciaccheri, Studi di ornati alcuni dei quali del Nasoni, S. III.6.

${ }^{16}$ Siena, Biblioteca degli Intronati, Ciaccheri, Disegni di Vincenzo Ferrati, S. II.1, $14 \mathrm{v}-15 \mathrm{r}$ e $22 \mathrm{v}$. 
gli, stilisticamente simili, rivelano l'abilità di cogliere la soluzione quadraturistica d'insieme, dando contemporaneamente attenzione ai particolari dell'ornato. La carica decorativa visibile nelle pitture della Sé di Oporto e di Lamego trova in questi anni una delle sue principali fonti d'origine.

La pastosità degli affreschi di Orlandi, capace di dare alle volute, ai fogliami, alle cartelle la consistenza dello stucco viene unita, da Nasoni, allo sguardo sintetico sull'intera tradizione quadraturistica e scenografica bolognese. A Oporto, negli sguanci di finestroni del presbiterio della Cattedrale, l'artista toscano è in grado, così, di recuperare contemporaneamente diversi modelli: la ripercussione in profondità di uno stesso elemento architettonico e l'organizzazione 'per angolo' propri del lessico bibienesco; la strutturazione chiara dello spazio architettonico dipinto attraverso l'uso di possenti colonne di marmo verde, che ricordano quelle affrescate dagli allievi di Agostino Mitelli nella chiesa di San Francesco in Rocca a Sassuolo; l'articolazione dello spazio stesso secondo modalità riscontrabili nelle opere di Stefano Orlandi; la matericità tattile conferita agli ornati dalla generazione di artisti emiliani attivi nelle prime decadi del Settecento.

A Bologna, lo studio e la preparazione in campo architettonico è mediato dall'arte della quadratura. Nella città emiliana, l'architettura è, prima di tutto, un modo di pensare lo spazio. In questo consiste l'eredità principale lasciata da Agostino Mitelli, il cui uso dell'affresco rappresentò la libertà di immaginare e 'costruire' nuove e coerenti spazialità architettoniche ${ }^{17}$. Il fatto che un quadraturista-scenografo si converta in architetto, non occasionalmente o per forza delle circostanze, ma per implicita preparazione è un dato acquisito nella formazione emiliana. Niccolò Nasoni trascorre a Bologna anni fondamentali. Non è più un giovane appren-

\footnotetext{
${ }^{17} \mathrm{Su}$ questo importante aspetto dell'arte di Agostino Mitelli vedi Ilusionismos. Os Tetos Pintados do Palácio Alvor, pp. 11-22.
} 
dista, dato che vi soggiorna tra i 27 e i 30 anni. E' dunque un artista maturo che sceglie di perfezionare la sua arte con cognizione di causa. Anche il trasferimento a Malta avviene grazie alla sua presenza a Bologna. La concatenazione degli avvenimenti è conosciuta attraverso le memorie di un più giovane pittore, Giovanni Zanardi, apprendista presso Stefano Orlandi al tempo della richiesta di un quadraturista, avanzata nel 1721 da "Sua Ecc.a Sig.r Commendatore Sig.r Pieri"18 il quale, come scrive Zanardi nella sua autobiografia manoscritta:

avea commissione di ritrovare un giovine per dovere mandare à dipingiere la Gran Chiesa di S. Giov. Batt.a in Malta con grosso stipendio e terminata l'opera se ritornare volea al suo paese gli sarebbe stato rimesso con una pensione annoa e col titolo di cavagliere servente [...] e si rivolgono al sud ${ }^{\circ}$ Nasoni con consenso del S.r Orlandi, furono stabilite le massime e si fece il contratto e da lì a poco partì per la sud.a opera. ${ }^{19}$

Il "cavaliere cittadino di Bologna"20 inviato dall'Ordine dei Cavalieri di Malta, di cui in altro punto del manoscritto Zanardi esplicita il nome "Pieri", si ritrova nominato come "cavaliere Girolamo Pieri" tra i membri del Collegio di Balia di Siena che, sul finire del 1721, incarica Nasoni, già di ritorno a Siena, di progettare il catafalco per le esequie della gran duchessa Marguerite-Louise d'Orléans-Medici' ${ }^{21}$. Nell'autobiografia di Zanardi l'episodio è narrato con un certo risentimento per non essere stato prescelto, ma l'incontro avvenuto

${ }^{18}$ Bologna, BCAB, Vita del Sig. Giovanni Bernardo Melchiorre Zanardi [...] scritta di proprio pugno, ms. B 95, c. 70 bis.

${ }^{19}$ Bologna, BCAB, Vita del Sig. Giovanni Bernardo Melchiorre Zanardi [...] scritta di proprio pugno, ms. B 95, c. 56. Nel manoscritto si trova anche un duplicato in "bella copia", ivi, cc. 320-332v.

${ }^{20}$ Bologna, BCAB, Vita del Sig. Giovanni Bernardo Melchiorre Zanardi [...] scritta di proprio pugno, ms. B 95, c. 56.

${ }^{21}$ Niccolò Nasoni (1691-1773), p. 44. 
nella scuola di Orlandi, in realtà, riflette la chiara volontà da parte dei cavalieri senesi dell'Ordine di Malta di dotarsi di esperti quadraturisti, i quali, certamente non ignoravano il periodo di perfezionamento di Nasoni, in quanto progettista nel 1715 del già ricordato arco trionfale e dei carri allegorici del 1720. Il contratto stilato a Bologna non può essere stato, come racconta Zanardi, un fatto fortuito, bensì la conseguenza del riconoscimento del prestigio di cui l'artista godeva nel contesto politico e artistico senese e la cui preparazione era in grado, a queste date, di eseguire l'importante committenza decisa da Marcantonio Zondadari, eletto alla guida del potente Ordine di Malta.

Nell'isola, infatti, Nasoni affronta impegnative imprese decorative. Tra il 1723 e il 1725 realizza gli affreschi nel palazzo Magistrale (tre corridoi, la cappella dell'appartamento invernale, la sala del consiglio), nel salone della Cancelleria e nella chiesa di san Giovanni ${ }^{22}$. Esercita dunque esclusivamente nell'ambito della pittura monumentale di quadratura, non dedicandosi all'attività scenografica e neppure cimentandosi in quella architettonica. Dal racconto di Giovanni Zanardi le condizioni del contratto contemplavano la possibilità di "ritornare al paese" 23 , una volta terminata l'opera. Le rocambolesche circostanze che spingono l'artista a lasciare l'isola sono note e ultimamente approfondite dagli studi di Giovan Battista Tedesco ${ }^{24}$. La scelta di trasferirsi in Portogallo, però, potrebbe essere stata presa anche sulla base di considerazioni professionali e non solo come scappatoia giudiziaria. Così come era accaduto con i membri della famiglia ChigiZondadari di Siena, l'apprezzamento, il potere e la politica

\footnotetext{
${ }^{22}$ Robert Smith, Nicolau Nasoni arquitecto do Porto, Lisboa, Livros Horizonte, 1966, pp. 33-35; Niccolò Nasoni (1691-1773), p. 31.

${ }^{23}$ Bologna, BCAB, Vita del Sig. Giovanni Bernardo Melchiorre Zanardi [...] scritta di proprio pugno, ms. B 95, c. 56.

${ }^{24}$ Vedi Giovanni Battista Tedesco, Nicolau Nasoni: formação de um pintor e de um artista da arte efémera em Itália (1691-1723).
} 
artistica promossa da alcuni esponenti della famiglia Távora e Noronha può aver offerto allettanti prospettive al pittore.

I primi anni Venti del Settecento rappresentano il momento di massima apertura artistica presso la corte joanina, dove la quadratura aveva riscosso grande successo grazie all'operato del fiorentino Vincenzo Bacherelli, attivo a Lisbona tra il 1701 e il 1721; confermato anche dalla scelta di affidare al quadraturista portoghese António Simóes Ribeiro la realizzazione tra il 1723 e il 1724 dei tre soffitti dipinti della biblioteca dell'Università di Coimbra ${ }^{25}$. Il dinamismo della capitale portoghese si specchia nelle vicende del capitolo della cattedrale portuense, che rimane sede vacante dal 1716, anno della nomina del vescovo, D. Tomás de Almeida, a patriarca di Lisbona. In questo periodo si distacca la politica artistica promossa dal decano D. Jerónimo de Távora e Noronha, fratello maggiore del vice-cancelliere dell'ordine dei cavalieri di Malta, D. Roque de Távora e Noronha, colui che propizia l'arrivo di Niccolò Nasoni da Malta a Oporto. Anche il loro fratello minore, Vicente de Távora e Noronha, entrerà a far parte del medesimo Ordine, ricoprendo la carica di ambasciatore di D. Antonio Manoel de Vilhena presso la corte del re Giovanni V.

Sin dall'inizio degli anni Venti, l'ambizione del monarca di porre alla guida dei Cavalieri di Malta un portoghese è

${ }^{25}$ Ho riflettuto sulla politica artistica di D. João V durante la prima metà del suo regno, proponendo una nuova interpretazione critica della complessa dinamica artistica in "La circolazione delle opere della stamperia De Rossi in Portogallo", Studio dell'Architettura Civile. Gli atlanti di architettura moderna e la diffusione dei modelli romani nell'Europa del Settecento, a cura di Aloisio Antinori, Roma, Quasar, 2013, pp. 143-163 (vedi anche per bibliografia precedente); "Filippo Juvarra a Lisbona: due progetti per un teatro regio e una complessa questione musicale", Filippo Juvarra (16781736). Architetto dei Savoia, Architetto in Europa. Atti del Congresso, Torino 2011, a cura di C. Ruggero (in corso di stampa); "Italia \& Portogallo: un incrocio di sguardi sull'arte della quadratura”, Di buon affetto e commerzio. Relaçôes luso-italianas na Idade Moderna, a cura di Nunziatella Alessandrini, Mariagrazia Russo, Gaetano Sabatini, Antonella Viola, Lisboa, CHAM, FCSH UNL, UAc, 2012, pp. 177-211. 
segnalata dalle lettere del nunzio apostolico. Esse registrano un certo disappunto nel 1720 e un aperto giubilo per quella di Vilhena nel 1722. Per l'elezione di Zondadari, il re, infatti, si congratula con il nunzio, affermando "che era amante al sommo degli italiani e in ispecie dei senesi, avendo goduto assai dell'elezione a Gran Maestro di un personaggio così grande e meritevole"26, aggiungendo però che aveva dato ordine ai suoi cavalieri portoghesi che:

stessero uniti al fine di far cadere l'elezione su un suo suddito e havendo questi operato tutto il contrario ne voleva fare pubblica dimostrazione castigandoli severamente. Aggiunse che altre volte haveano fatte somigliante vigliaccheria e che lui solo conosceva molto bene il naturale de suoi sudditi, pieni di un'ambiziosa disunione dicendone molti improperii. ${ }^{27}$

\section{Nel 1722 i fatti corrono diversamente e:}

Colle lettere di Malta fu ricevuto da questo Sig.r D. Lopo de Almeyda, ricevitore della religione gerosolimitana in questo regno, l'avviso della morte del Gran Maestro Marco Antonio Zondadari nel giorno 16 del mese passato [giugno] e che in suo luogo sia stato eletto D. Antonio Manoel de Vilhena portoghese, del quale successo non solo ne ha mostrato uno straordinario contento tutto il suo illustre parentado ma goduto altresì la corte tutta. ${ }^{28}$

Il coinvolgimento di potenti famiglie portoghesi nella politica artistica del regno è un dato fondamentale per comprendere il dinamismo di questo periodo. I Távora e Noronha ricoprono incarichi di primo piano all'interno della diocesi di Oporto e esercitano un forte potere nelle alte gerarchie

\footnotetext{
${ }^{26}$ Archivio Segreto Vaticano, Segr. Stato, Portogallo, 75, c. 440v.

${ }^{27} \mathrm{Ib}$.

${ }^{28}$ ASV. Segr. Stato, Portogallo 78, c. 233. L'arrivo di una nave dei cavalieri di Malta e i sontuosi festeggiamenti sono descritti alle cc. 285-286.
} 
ecclesiastiche, strettamente vincolato anche agli interessi e alla forza dell'Ordine di Malta. In questo contesto l'arrivo di Nasoni assume l'aspetto di una chiamata consapevole da parte di Jerónimo de Távora e Noronha, piuttosto che di una via di fuga per l'artista.

Sin dall'elezione di D. Tomás de Almeida e Noronha a vescovo di Oporto (1709-1717) comincia un piano di progettazione architettonica e artistica che il Capitolo prosegue dopo la sua partenza per Lisbona. La costruzione della casa del capitolo e la realizzazione del soffitto cassetto nato, dipinto dall'italiano Giovanni Battista Pachini nel 1722, evidenziano questa volontà di rinnovamento, priva però di un linguaggio aggiornato e apprezzato non solo alla corte di Giovanni V ma internazionalmente in Europa. Nasoni era l'artista giusto e se dapprima, giunto in Portogallo, si dedica alla sua specialità, la quadratura, l'urgenza costruttiva lo spinge ad applicare le sue conoscenze al campo del costruito, operando uno slittamento che, come ho dimostrato sopra, non era un 'prestito' ma una vera e propria possibilità fornitagli dalla sua formazione in Italia. L'urgenza costruttiva si collega, ancora una volta, con la politica joanina e il controllo in campo religioso che il re si prefiggeva di strutturare, controllando i poteri del clero regolare e dando visibilità e centralità alla nuova dignità del patriarca. All'esterno il dialogo con il Vaticano si fa serrato e, spesso, conflittuale in questi anni. All'interno la riorganizzazione ecclesiastico-diocesana coinvolge direttamente la formazione e il ruolo dei chierici secolari, favorendo lo sviluppo e il consolidamento delle confraternite di São Pedro dos Clérigos. Questo movimento investe contemporaneamente i territori d'aquém e além-mar, determinando una stretta relazione di tipologia architettonica tra le chiese omonime erette in quegli anni a Recife, Rio de Janeiro e Oporto. L'uso simbolico della pianta ovale o ottagonale si unisce, a Oporto, ad un chiaro riferimento alla basilica di Mafra. Nel 1732 i confratelli registrano un 
pagamento di 240 reis per causa "de vir a planta de Mafra"29, proprio nel momento in cui Nasoni sta progettando la loro chiesa, la cui prima pietra è posta il 2 giugno 1732. Nel corso del tempo varie perizie modificano il progetto che, inizialmente prevedeva due torri campanarie e una cupola. Questi elementi, seppur posti in un edificio a pianta ovale e non basilicale, avrebbero creato un'evidente corrispondenza con il complesso di Mafra, simbolo dell'affermazione della politica joanina verso la Santa Sede ${ }^{30}$. Infatti, seppur il progetto risalga al 1716, la basilica di Mafra e l'impianto generale del palazzo-convento vennero effettivamente realizzati durante gli anni della rottura diplomatica tra la corte portoghese e quella pontificia. Com'è noto la basilica è conclusa e consacrata in due anni di lavori serrati (1729-1730), nonostante mancassero ancora la cupola e il coronamento delle due torri, nella cui costruzione intervenne anche l'architetto romano Antonio Canevari presente a Lisbona.

Niccolò Nasoni era l'artista che possedeva il linguaggio e le conoscenze adeguate per realizzare un edificio, il cui valore simbolico travalicava i confini cittadini e si inseriva in un progetto di affermazione politica di ben più vasto respiro. Di questa sua capacità dava saggio sin dalla posa della prima pietra quando, grazie all'arte dell'effimero, suggeriva visivamente la sontuosità che sarebbe stata poi realizzata in granito:

1732 - em dois do presente mes de junho [...] Fizerao no sitio em que se faz a da igreja huas luminarias com pintura de prespectiva, formando o frontespicio da igreja e mais de 6000 luzes [...] espera-ce que vista a planta e agradável sitio seja hum dos mais magestosos templos do reino ${ }^{31}$

${ }^{29}$ B. Xavier Coutinho, A igreja e a Irmandade dos Clérigos. Apontamentos para a sua história, Porto, Câmara Municipal do Porto, 1965, p. 95. Il documento citato dall'autore si trova nell'Arquivo da Irmandade dos Clérigos, Livro de Contas, c. 196.

${ }^{30}$ Giuseppina Raggi, "La circolazione delle opere della stamperia De Rossi in Portogallo".

${ }^{31}$ Biblioteca Nacional de Portugal, Manuscritos, col. Pombalina, cod. 672, c. 92. Documento citato da Coutinho 1965. 
Nella chiesa dos Clérigos il lessico borrominiano si sposa al gusto per l'impianto scenografico e il particolare decorativo che rendono manifesta la formazione toscana e, soprattutto, l'importanza artistica degli anni trascorsi a Bologna. Il perfezionamento nei vari campi d'applicazione della prospettiva esercitato da Nasoni nella città emiliana convergono, a Oporto, in un sapere architettonico capace di gestire al meglio le possibilità, e anche le difficoltà, del costruire. Se la torre campanaria, così come è celebrata oggi, non fu progettata sin dall'inizio, la soluzione adottata mostra l'intelligenza e la coerenza con cui Niccolò seppe rispondere alle sfide insorte durante i trent'anni necessari all'edificazione dell'intero complesso. L'altezza insolita della torre considera il dislivello su cui la chiesa è costruita e calcola le principali direttrici di visione, seppur oggi modificate dai nuovi tracciati viari aperti nella seconda metà del Settecento. Costruita tra il 1758 e il 1763 , la torre riassume le caratteristiche dell'arte portoghese di Nasoni. Il granito conferisce agli ornati una corposità peculiare che si ricollega a quel gusto materico già visibile nelle quadrature del presbiterio della Cattedrale. La struttura della torre è chiaramente leggibile al di sotto degli elementi decorativi e se la comparazione visiva con le vicine architetture di influenza inglese e neopalladiana le conferisce un'immagine tipicamente 'barocca', un'analisi attenta e una visione scevra dalle comuni dicotomie stilistiche evidenzia la ricchezza evocativa di modelli, stili e culture che, in essa, si coagulano. Diversamente da quanto affermò Alfonso Rodrigues Ceballos e sulla base del forte valore politico-simbolico che la costruzione della chiesa e della Torre dos Clérigos voleva rappresentare, l'unicità della torre di Niccolò Nasoni non è determinata dalla sua "fisionomia exotica [...] di carattere ispano-arabe" 32 bensì, al contrario, dal sapere declinare, in un linguaggio appropriato al tempo e al contesto locali, le dinamiche culturali e politiche di un'intera epoca.

\footnotetext{
${ }^{32}$ Alfonso Rodriguez Ceballos, La torre Nueva de G. B. Contini di Zaragoza y la torre de São Pedro dos Clérigos, de Nasoni, en Oporto, Braga, Câmara Municipal de Braga, 1974.
} 